\title{
Introduction of a Quality Improvement Bundle Is Associated with Reduced Exposure to Mechanical Ventilation in Very Preterm Infants
}

\author{
Stacey Chi-Yan Lo ${ }^{a}$ Risha Bhatia ${ }^{a, b}$ Calum T. Roberts ${ }^{a, b, c}$ \\ aDepartment of Paediatrics, School of Clinical Sciences at Monash Health, Monash University, Melbourne, VIC, \\ Australia; ${ }^{b}$ Monash Newborn, Monash Children's Hospital, Melbourne, VIC, Australia; 'The Ritchie Centre, Hudson \\ Institute of Medical Research, Melbourne, VIC, Australia
}

\section{Keywords}

Infant · Preterm · Mechanical ventilation · Pulmonary surfactant . Continuous positive airway pressure - Umbilical cord

\begin{abstract}
Introduction: Exposure to mechanical ventilation (MV) is a risk factor for bronchopulmonary dysplasia (BPD) in very preterm infants (VPTIs). We assessed the impact of a quality improvement (QI) bundle in VPTIs ( $<32$ week gestation) on exposure to MV. Methods: We introduced a QI bundle consisting of deferred cord clamping (DCC), nasal bubble continuous positive airway pressure (bCPAP) in the delivery room (DR), and minimally invasive surfactant therapy (MIST). We compared respiratory outcomes and neonatal morbidity in historical pre-QI (July-December 2017) and prospective postQI (February-July 2019) cohorts (QICs) of VPTIs. We pre-specified an adjusted analysis to account for the effects of gestational age, sex, antenatal steroids, and any demographic data that significantly differed between cohorts. $\boldsymbol{R e}$ sults: The pre-QI and post-QICs included 87 and 98 VPTIs, respectively. The post-QIC had decreased rates of MV in the DR (adjusted odds ratio [aOR] 0.26, 95\% confidence interval [Cl] 0.09-0.71), in the first $72 \mathrm{~h}$ of life (aOR $0.27,95 \% \mathrm{Cl} 0.11-$ 0.62 ) and during admission (aOR $0.28,95 \% \mathrm{Cl} 0.12-0.66$ ).
\end{abstract}

Rates of BPD, combined BPD/death, and BPD severity were similar. The post-QIC was less likely to be discharged with home oxygen (aOR $0.27,95 \% \mathrm{Cl} 0.08-0.91$ ). Necrotising enterocolitis grade $\geq 2$ increased (aOR 19.01, 95\% Cl 1.93-188.6) in the post-QIC. Conclusion: In this rapid-cycle QI study, implementation of a QI bundle consisting of DCC, early nasal bCPAP, and MIST in VPTIs was associated with reduced rates of $M V$ in the $D R$, in the first $72 \mathrm{~h}$ of life and during admission, and reduced need for home oxygen.

(c) 2021 The Author(s).

Published by S. Karger AG, Basel

\section{Introduction}

Bronchopulmonary dysplasia (BPD), defined as requirement for supplemental oxygen or respiratory support at 36 week corrected gestational age (CGA), is a significant complication of preterm birth, associated with adverse effects persisting into later life [1-4]. Exposure to mechanical ventilation (MV) is a significant risk factor for developing BPD in very preterm infants (VPTIs) born before 32 week gestation [5]. Advances in perinatal care have improved the survival of VPTIs without reduction in the incidence of BPD $[6,7]$.
(C) 2021 The Author(s)

Published by S. Karger AG, Basel

This is an Open Access article licensed under the Creative Commons Attribution-NonCommercial-4.0 International License (CC BY-NC) (http://www.karger.com/Services/OpenAccessLicense), applicable to the online version of the article only. Usage and distribution for commercial purposes requires written permission.
Correspondence to:

Stacey Chi-Yan Lo, staceylocy@ gmail.com

Calum T. Roberts, calum.roberts@monash.edu 
Previous studies reporting the outcomes of quality improvement (QI) initiatives have shown mixed results. Neonatal intensive care units (NICUs) have reported reductions in MV and BPD, in small cohorts ranging from 107 to 314 infants [8-10]. Whilst the specific QI interventions varied, common practices included adopting a multidisciplinary team, promoting early continuous positive airway pressure (CPAP) over MV, implementing uniform intubation and extubation criteria, and regular debriefing and feedback during QI implementation [8-10]. Similar studies observed reduced rates of delivery room (DR) intubation after implementing DR bundles, without any change in BPD incidence, or without reduction in either MV or BPD [11-13].

Many QI studies have focused on CPAP and intubation criteria to decrease MV and BPD rates, but the impact of the more recent interventions, such as deferred cord clamping (DCC), and minimally invasive surfactant therapy (MIST), have not been assessed. Randomised controlled trials and meta-analyses have reported reduced in-hospital mortality in infants who received DCC and reductions in $\mathrm{BPD}$ and combined $\mathrm{BPD} /$ death in infants who received MIST $[14,15]$.

A face mask with T-piece device is the most commonly used CPAP interface in the DR but has limitations such as mask leak and airway obstruction [16-18]. To mitigate these, alternative approaches may be used, such as bubble CPAP (bCPAP), applied via the nasal interface. bCPAP is often favoured over ventilator-derived CPAP due its lower cost, increased portability, and potential for improved clinical outcomes [11,19]. Although existing evidence for bCPAP comes from NICU practice, there is no obvious reason why it cannot be used effectively in the DR. We aimed to assess the effect of the introduction of a QI bundle consisting of DCC, early nasal bCPAP in the DR, and MIST, on respiratory outcomes of VPTIs at a tertiary NICU in Victoria, Australia.

\section{Materials and Methods}

\section{Setting}

Monash Newborn at Monash Children's Hospital (MCH) is the largest NICU in Victoria, Australia, with $>1,500$ admissions annually. Deliveries of VPTIs are attended by a neonatal resuscitation team, consisting of a neonatal registrar and/or fellow, a nurse trained in neonatal resuscitation, and, at $<26$ week gestation, a consultant neonatologist. Resuscitation or stabilisation of the infant is performed in the DR and details recorded on a scribe sheet.

Prior to introduction of the QI bundle, infants received immediate umbilical cord clamping as standard care. VPTIs were com-

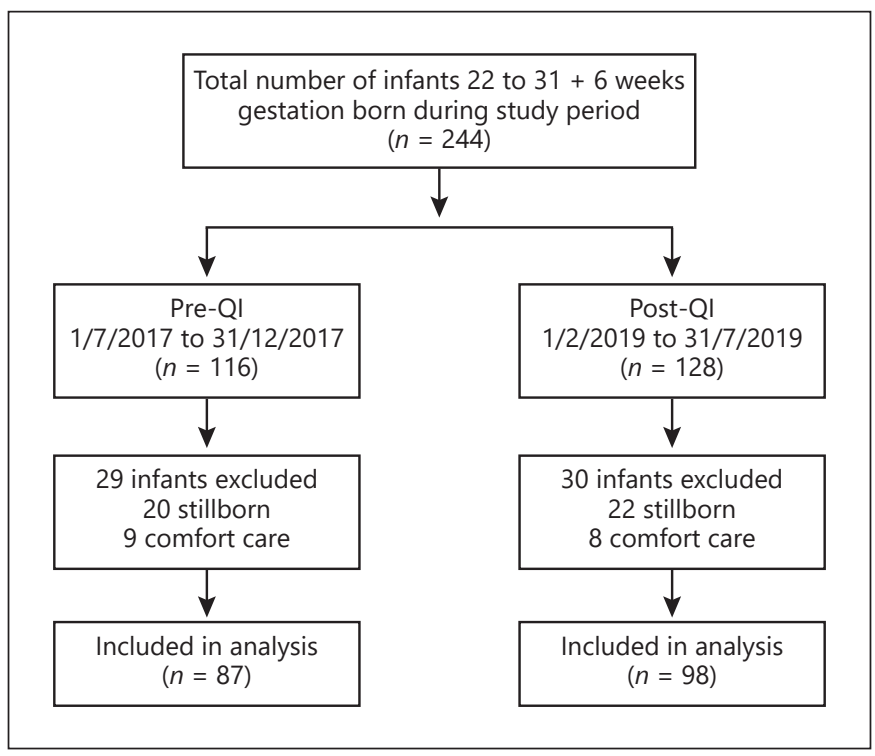

Fig. 1. Screening of eligible births during the study period.

menced on CPAP or positive pressure ventilation via a T-piece resuscitator and face mask (Neopuff ${ }^{\mathrm{TM}}$; Fisher and Paykel, Auckland, New Zealand). The decision to intubate was left largely at the discretion of the attending clinician, with no specific indications stated within local guidelines. Infants who did not require intubation were maintained on face mask CPAP during stabilisation and transitioned to nasal CPAP via a transport ventilator for transportation to the NICU. Infants were intubated for surfactant administration if oxygen requirement exceeded $40 \%$.

\section{Planning and Implementation of the QI Bundle}

In 2017, we identified BPD as a priority for improvement. Following review of existing practice, reducing exposure to MV was identified as the primary driver to reduce BPD incidence. Optimising transition at birth and avoiding intubation, unless necessary, were identified as secondary drivers through which this could be achieved. A QI bundle consisting of DCC after establishment of spontaneous breathing, emphasis on non-invasive ventilation by optimising bCPAP as the initial mode of respiratory support in the DR, and MIST for infants on CPAP requiring surfactant was introduced in 2018 (online suppl. Table 1; for all online suppl. material, see www.karger.com/doi/10.1159/000518392).

A staged introduction of each intervention and ongoing education to maintain medical and nursing competence was implemented. Guidelines were made available on the staff intranet, and copies were attached to neonatal resuscitation trolleys in birth suites and theatres. By January 2019, all interventions had been implemented into routine practice.

\section{Study Population}

In keeping with a rapid cycle QI approach, we compared process measures and associated clinical outcomes in VPTIs born in the immediate 6-month period before and after implementation of the QI bundle into routine practice. Infants born between July 1 
Table 1. Maternal and neonatal characteristics

\begin{tabular}{|c|c|c|c|c|c|}
\hline \multirow[b]{2}{*}{ ANS, any } & \multicolumn{2}{|c|}{ Pre-QI $(n=87)$} & \multicolumn{2}{|c|}{ Post-QI $(n=98)$} & \multirow{2}{*}{$\frac{p \text { value }}{\mathbf{0 . 0 5}^{*}}$} \\
\hline & 84 & $(96.6)$ & 87 & $(88.8)$ & \\
\hline Complete course & 61 & $(70.1)$ & 70 & $(71.4)$ & \\
\hline Incomplete course & 23 & $(26.4)$ & 17 & $(17.3)$ & 0.07 \\
\hline None & 3 & $(3.4)$ & 11 & $(11.2)$ & \\
\hline Magnesium sulphate, any & 51 & $(58.6)$ & 66 & $(67.3)$ & 0.22 \\
\hline Complete course & 51 & $(58.6)$ & 61 & $(62.2)$ & \\
\hline Incomplete course & 0 & $(0.0)$ & 5 & $(5.1)$ & 0.06 \\
\hline None & 36 & $(41.4)$ & 32 & $(32.7)$ & \\
\hline Pre-eclampsia & 17 & $(19.5)$ & 17 & $(17.3)$ & 0.70 \\
\hline GDM & 26 & $(29.9)$ & 12 & $(12.2)$ & $<0.01^{*}$ \\
\hline $\mathrm{APH}$ & 28 & $(32.2)$ & 18 & $(18.4)$ & $0.03 *$ \\
\hline Chorioamnionitis & 17 & $(19.5)$ & 16 & $(16.3)$ & 0.57 \\
\hline \multicolumn{6}{|l|}{ Delivery mode } \\
\hline Vaginal & 36 & $(41.4)$ & 45 & $(45.9)$ & \multirow{2}{*}{0.54} \\
\hline Emergency caesarean & 51 & $(58.6)$ & 53 & $(54.1)$ & \\
\hline Male sex & 42 & $(48.3)$ & 52 & $(53.1)$ & 0.52 \\
\hline GA & 29.1 & $(27,30.9)$ & 29.7 & $(27.4,30.9)$ & 0.48 \\
\hline Extremely preterm infant ( $<28$ week gestation) & 28 & $(32.2)$ & 27 & $(27.6)$ & 0.49 \\
\hline Gestation $<25$ weeks & 5 & $(5.8)$ & 6 & $(6.1)$ & 0.91 \\
\hline Birth weight, g, mean, SD & 1,240 & $(415)$ & 1,217 & $(405)$ & 0.70 \\
\hline Extremely low birth weight $(<1,000 \mathrm{~g})$ & 26 & $(29.9)$ & 36 & $(36.7)$ & 0.33 \\
\hline Multiple birth & 20 & $(23.0)$ & 23 & $(23.5)$ & 0.94 \\
\hline Fetal distress prior to birth & 57 & $(65.5)$ & 59 & $(60.2)$ & 0.46 \\
\hline Congenital abnormality (any) & 12 & $(13.8)$ & 13 & $(13.3)$ & 0.92 \\
\hline Major congenital abnormality & 1 & $(1.1)$ & 2 & $(2.0)$ & 0.63 \\
\hline
\end{tabular}

All data are displayed as $n(\%)$ or median (IQR) unless otherwise stated. QI, quality improvement; GA, gestational age; ANS, antenatal steroid; GDS, gestational diabetes; APH, antepartum haemorrhage.

and December 31, 2017 constituted the retrospective pre-QI cohort (QIC), and infants born between February 1 and July 31, 2019 constituted the prospective post-QIC. Infants who were stillborn or who received comfort care, rather than active resuscitation and intensive care at birth, were excluded from the study.

\section{Process Measures and Outcomes}

The primary outcome was exposure to MV, measured at 3 time points: in the DR, in the first $72 \mathrm{~h}$ of life, and during NICU admission. Prior to commencing the study, we performed a power calculation, which indicated that a sample of 100 infants per group (estimated admissions during a 6-month period) would provide $80 \%$ power to detect reduction in MV during admission from $48 \%$ (unpublished unit data for calendar year 2016) to $29 \%$. Secondary outcomes included duration of $\mathrm{MV}$, incidence of $\mathrm{BPD}$, and combined $\mathrm{BPD} /$ death. $\mathrm{BPD}$ was defined as requirement for respiratory support or oxygen at or after 36 week CGA, excluding infants who required brief elective respiratory support, for example, for surgical procedures. Process measures included documented DCC, maximum mode of DR respiratory support, trial of CPAP prior to $\mathrm{MV}$, and mode of surfactant administration. Balancing measures were non-respiratory morbidity and mortality.

\section{Data Collection and Analysis}

One team member (SL) collected data from electronic medical records for all infants. Analysis was performed using SPSS Statistics Version 26.0 (Armonk, NY, USA: IBM Corp). For all tests, a $p$-value $<0.05$ (2-sided) was considered significant. Categorical variables were compared using Pearson's $\chi^{2}$ test (cells $>5$ subjects) or Fisher's exact test (cells $<5$ subjects). Ordinal categorical outcomes were compared with linear-by-linear association (CochranArmitage test). For dichotomous outcomes, odds ratios were reported with $95 \%$ confidence intervals ( $95 \%$ CIs). Continuous variables were compared with the independent samples $T$-test and displayed as mean \pm standard deviation, or with the Mann-Whitney $U$ test and displayed as median and interquartile range, as appropriate.

Prior to data collection, an adjusted analysis was planned to account for differences in demographic variables that could potentially influence key outcomes. GA, antenatal steroid (ANS) administration, and sex were pre-specified for inclusion in this adjustment, with the planned addition of any further demographic variables that significantly differed between the 2 cohorts. Logistic regression analysis was performed and dichotomous outcomes compared using adjusted odds ratios (aORs). 
Table 2. Comparison of DR interventions and outcomes

\begin{tabular}{|c|c|c|c|c|c|}
\hline \multirow[b]{2}{*}{ Documented DCC } & \multicolumn{2}{|c|}{ Pre-QI $(n=87)$} & \multicolumn{2}{|c|}{ Post-QI $(n=98)$} & \multirow{2}{*}{$\begin{array}{l}p \text { value } \\
<0.01^{*}\end{array}$} \\
\hline & 0 & $(0.0)$ & 40 & $(40.8)$ & \\
\hline \multicolumn{6}{|l|}{ Maximum DR respiratory support } \\
\hline Intubation & 23 & $(26.4)$ & 12 & $(12.2)$ & $0.01^{*}$ \\
\hline Face mask IPPV & 51 & $(58.6)$ & 51 & $(52.0)$ & 0.37 \\
\hline CPAP & 13 & $(14.9)$ & 32 & $(32.7)$ & $<0.01^{*}$ \\
\hline None & 0 & $(0.0)$ & 3 & $(3.1)$ & 0.10 \\
\hline Face mask IPPV, any & 73 & $(83.9)$ & 63 & $(64.3)$ & $<0.01^{*}$ \\
\hline CPAP, any & 72 & $(82.8)$ & 88 & $(89.8)$ & 0.16 \\
\hline Surfactant, any & 37 & $(42.5)$ & 36 & $(36.7)$ & 0.42 \\
\hline ETT, any & 37 & $(42.5)$ & 23 & $(23.5)$ & $<0.01^{*}$ \\
\hline MIST, any & 0 & $(0.0)$ & 19 & $(19.4)$ & $<0.01^{*}$ \\
\hline Both & 0 & $(0.0)$ & 6 & $(6.1)$ & $0.02 *$ \\
\hline Age of first surfactant, days & 1 & $(1,1)$ & 1 & $(1,1)$ & 0.21 \\
\hline \multicolumn{6}{|l|}{ Surfactant doses, $n$} \\
\hline 1 & 28 & $(32.2)$ & 18 & $(18.4)$ & \\
\hline 2 & 8 & $(9.2)$ & 16 & $(16.3)$ & 0.12 \\
\hline $3+$ & 1 & $(1.1)$ & 2 & $(2.0)$ & \\
\hline 1 min APGAR & 5 & $(4,7)$ & 7 & $(5,8)$ & $0.01 *$ \\
\hline 5 min APGAR & 8 & $(7,9)$ & 8 & $(7,9)$ & 0.28 \\
\hline Intubation in DR & 23 & $(26.4)$ & 12 & $(12.2)$ & $0.02^{*}$ \\
\hline Intubation without trial of CPAP & 15 & $(17.2)$ & 7 & $(7.1)$ & 0.03* \\
\hline Age at intubation, $\min ^{1}$ & 9 & $(5,12)$ & 7 & $(7,24)$ & 0.89 \\
\hline Chest compressions & 4 & $(4.6)$ & 4 & $(4.1)$ & 0.86 \\
\hline Adrenaline & 0 & $(0.0)$ & 1 & $(1.0)$ & 0.35 \\
\hline Admission age, $\min$ & 29 & $(24,37)$ & 28 & $(24,36)$ & 0.41 \\
\hline Admission temperature, ${ }^{\circ} \mathrm{C}$ & 36.6 & $(36.2,37.0)$ & 36.5 & $(35.9,37)$ & 0.13 \\
\hline Hypothermia $\left(<36^{\circ} \mathrm{C}\right)$ & 14 & $(16.1)$ & 26 & $(26.5)$ & 0.08 \\
\hline Hyperthermia $\left(>38^{\circ} \mathrm{C}\right)$ & 4 & $(4.6)$ & 1 & $(1.0)$ & 0.17 \\
\hline
\end{tabular}

All data are displayed as $n$ (\%) or median (IQR). DCC, deferred cord clamping; IPPV, intermittent positive pressure ventilation; CPAP, continuous positive airway pressure; ETT, endotracheal tube; MIST, minimally invasive surfactant therapy; QI, quality improvement; aOR, adjusted odds ratio; DR, delivery room. ${ }^{1}$ Of infants who were intubated.

\section{Results}

There were 244 infants between 22 and $31+6$ week gestation born during the 2 study periods (Fig. 1). After excluding infants who were stillborn or received comfort care, a total of 185 infants were included: 87 in the preQIC and 98 in the post-QIC. Three maternal and neonatal characteristics differed between groups (Table 1) and were included in the adjusted analysis: lower rates of ANS, gestational diabetes, and antepartum haemorrhage were observed in the post-QIC.

There were significant differences in DR practices following introduction of the QI bundle (Table 2). Infants in the post-QIC were more likely to receive DCC and to receive $\mathrm{CPAP}$ as the maximum mode of respiratory support in the DR, and less likely to be administered intermittent positive pressure ventilation. Nineteen infants in the post-QIC received surfactant via MIST, and there was a concurrent decrease in endotracheal tube surfactant administration.

Rates of MV decreased in the post-QIC (Table 3). The OR for intubation remained lower after adjustment for GA, ANS, sex, antepartum haemorrhage, and gestational diabetes. In the DR, fewer infants in the post-QIC were intubated both overall (aOR 0.26, 95\% CI 0.09-0.71) and without a trial of CPAP (aOR 0.30, 95\% CI 0.09-0.99). There were also decreases in MV in the first $72 \mathrm{~h}$ of life (aOR 0.27, 95\% CI 0.11-0.62) and during admission (aOR $0.28,95 \%$ CI $0.12-0.66)$ in the post-QIC. Whilst the total duration of MV did not significantly differ, infants in the post-QIC were less likely to require MV after 36 week CGA. The post-QIC were more likely to receive nasal high 
Table 3. NICU respiratory interventions and outcomes

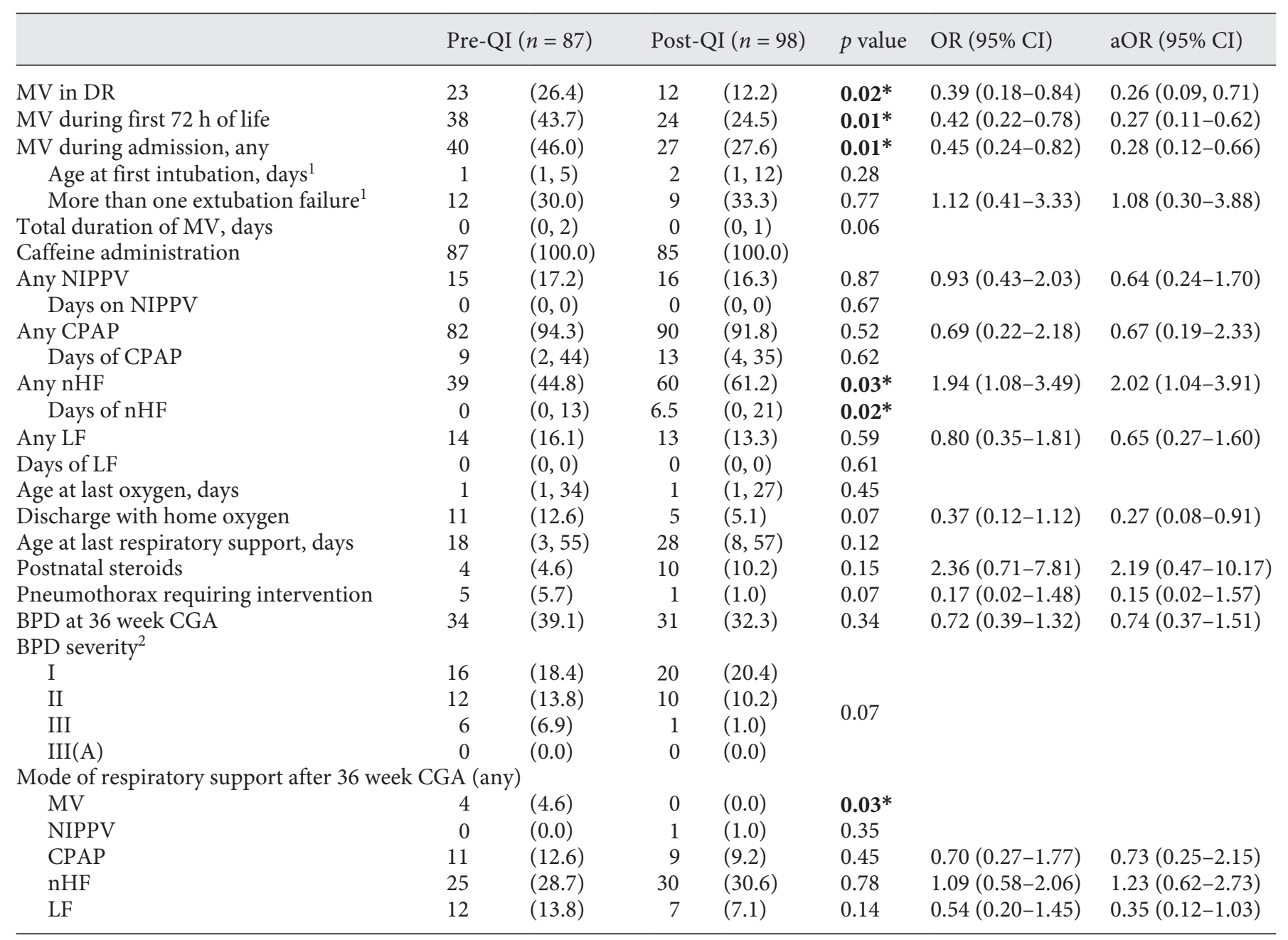

All data are displayed as $n$ (\%) or median (IQR). BPD, severity as per the NICHD Workshop definition of BPD, 2018 [20]. NIPPV, nasal intermittent positive pressure ventilation; CPAP, continuous positive airway pressure; nHF, nasal high flow; LF, low flow oxygen; $\mathrm{BPD}$, bronchopulmonary dysplasia; CGA, corrected gestational age; MV, mechanical ventilation; QI, quality improvement; NICU, neonatal intensive care unit; DR, delivery room; CI, confidence interval. ${ }^{1}$ Of infants who were ventilated. ${ }^{2}$ Linear-by-linear association test performed for $p$ value.

flow during admission (aOR 2.02, 95\% CI 1.04-3.91) and for longer duration but were less likely to be discharged on home oxygen (aOR 0.27 , 95\% CI 0.08-0.91).

There were no differences in incidence of BPD (aOR $0.74,95 \%$ CI $0.37-1.51$ ), death before discharge (aOR 1.09 , 95\% CI 0.36-4.54), or combined BPD/death (aOR $0.94,95 \%$ CI $0.45-1.96)$. Infants in the post-QIC had an increased incidence of necrotising enterocolitis (NEC) of grade 2 and above (aOR 19.01, 95\% CI 1.93-188.6) and decreased age of first full enteral feeds (Table 4). NEC of grade 2 or above is an outcome routinely recorded for contribution to the Australian and New Zealand Neonatal
Network (ANZNN). Given the unexpected increase in the post-QIC, we compared the rates for all infants $<32$ week gestation admitted to our unit in the calendar years 2018 and 2019 , finding no statistically significant difference: $4 / 230(1.7 \%)$ versus $10 / 211(4.7 \%)$, respectively, $p=0.10$.

\section{Discussion}

A QI bundle consisting of DCC, early nasal bCPAP, and MIST was associated with significantly decreased rates of $\mathrm{MV}$ in the $\mathrm{DR}$, the first $72 \mathrm{~h}$ of life, and during 
Table 4. Neonatal non-respiratory morbidity and mortality

\begin{tabular}{|c|c|c|c|c|c|}
\hline & Pre-QI $(n=87)$ & Post-QI $(n=98)$ & $p$ value & OR $(95 \% \mathrm{CI})$ & $\mathrm{aOR}(95 \% \mathrm{CI})$ \\
\hline Death before discharge & $5(5.7)$ & $6(6.1)$ & 0.91 & $1.07(0.31-3.64)$ & $1.09(0.36-4.54)$ \\
\hline Combined BPD or death & $37(42.5)$ & $37(38.5)$ & 0.58 & $0.82(0.45-1.48)$ & $0.94(0.45-1.96)$ \\
\hline PDA & $18(20.7)$ & $20(20.4)$ & 0.96 & $0.98(0.48-2.01)$ & $1.15(0.46-2.90)$ \\
\hline \multicolumn{6}{|l|}{ Treatment } \\
\hline Medical & $6(6.9)$ & $13(13.3)$ & & & \\
\hline Surgical & $2(2.3)$ & $0(0.0)$ & 0.17 & & \\
\hline None & $10(11.5)$ & $7(7.1)$ & & & \\
\hline Culture-positive sepsis & $15(17.2)$ & $16(16.3)$ & 0.87 & $0.94(0.43-2.03)$ & $0.88(0.35-2.23)$ \\
\hline Blood & $15(17.2)$ & $16(16.3)$ & 0.87 & $0.94(0.43-2.03)$ & $0.88(0.35-2.23)$ \\
\hline CSF & $1(1.1)$ & $1(1.0)$ & 0.93 & $0.89(0.05-14.39)$ & $1.16(0.06-21.7)$ \\
\hline NEC (stage 2 or higher) & $1(1.1)$ & $10(10.2)$ & $<0.01^{*}$ & $9.77(1.22-77.9)$ & $19.01(1.93-188.6)$ \\
\hline $\mathrm{ROP}$, any & $12(13.8)$ & $16(16.3)$ & 0.63 & $1.22(0.54-2.75)$ & $1.47(0.54-4.04)$ \\
\hline Laser treatment & $2(2.3)$ & $2(2.0)$ & 0.90 & $0.88(0.12-6.42)$ & $0.62(0.03-11.2)$ \\
\hline Severe IVH (grade 3-4) & $3(3.4)$ & $3(3.1)$ & 0.88 & $0.88(0.17-4.50)$ & $0.52(0.08-3.53)$ \\
\hline PVL & $2(2.3)$ & $2(2.0)$ & 0.90 & $0.88(0.12-6.42)$ & $0.70(0.06-7.85)$ \\
\hline Age at first full enteral feeds, days ${ }^{1}$ & $13(9,18)$ & $10(8,13)$ & $0.02 *$ & & \\
\hline Duration of tertiary hospital admission & $37(14,79)$ & $23(20,66)$ & 0.95 & & \\
\hline
\end{tabular}

All data are displayed as $n(\%)$ or median (IQR). ${ }^{*} p<0.05$. BPD, bronchopulmonary dysplasia; PDA, patent ductus arteriosus; CSF, cerebrospinal fluid; NEC, necrotising enterocolitis; ROP, retinopathy of prematurity; IVH, intraventricular haemorrhage; PVL, periventricular leucomalacia; QI, quality improvement; aOR, adjusted odds ratio; CI, confidence interval. ${ }^{1}$ Five infants who died prior to receiving enteral feeds were excluded from the analysis (2 pre-QI, 3 post-QI).

admission. These results may be attributable to the interventions in the QI bundle, and a culture shift towards decreased dependence on intubation and increased use of non-invasive support. Following the introduction of MIST, we also observed a decrease in surfactant administration via an endotracheal tube. A meta-analysis found that DCC does not reduce rates of DR intubation but did report reduced in-hospital mortality [14]. We did not observe this in our study, but our sample size is too small to detect such a difference. However, the higher 1-min APGAR score seen in the post-QIC may indicate improved cardiorespiratory stability associated with DCC. Although the recorded rate of DCC post-QI was lower than expected (40.8\%), this may reflect that some infants received DCC, but this was not documented. We have since revised our DR documentation sheet to include mandatory documentation of DCC. However, some infants may have met the criteria for early cord clamping (online sup$\mathrm{pl}$. Table 1). Although the admission temperature did not differ between groups, hypothermia $<36^{\circ} \mathrm{C}$ was relatively common $(26.5 \%$ in the post-QI group), and additional measures to maintain normothermia during DCC, stabilisation, and transport may be of benefit.

We observed an increase in CPAP as the maximum mode of DR respiratory support in the post-QIC; both early CPAP and MIST have been associated with reduced rates of MV in keeping with large randomised controlled trials [20-23]. Decreases in DR intubation following implementation of respiratory QI bundles incorporating early nasal CPAP, rather than routine intubation, have resulted in significant reductions in MV [8-12]. Studies have shown bCPAP to have lower resistance and imposed work of breathing than T-piece CPAP, and the oscillatory effect it provides may produce higher pressure $[20,24]$. We routinely apply bCPAP in the DR using a nasal mask, a very low resistance interface that may enhance pressure transmission [21].

Despite reduced rates of MV in the post-QIC, we did not observe differences in the incidence of BPD, death, or combined BPD/death. Our sample size is likely too small to detect a difference in BPD. As the pathogenesis of BPD is multifactorial, an improvement in other domains such as ANS administration may be required. These results are similar to other single-centre QI studies reporting decreased rates of intubation and MV following QI initiatives, without effect on BPD incidence $[11,12]$.

Three single-centre QI initiatives have observed reductions in BPD in their post-QIC of very low-birth weight infants [8-10]. These studies identified very high compliance to their QI bundles. They attributed this to 
being smaller centres with cohesive teams, facilitating greater engagement, targeted training, and regular feedback and debriefing. Such high compliance to major practice changes may be less easily achievable over a short time span in larger centres such as $\mathrm{MCH}$; reports from larger NICUs and collaborative studies have shown less impact on BPD rates [25-27].

Fewer infants in the post-QIC were discharged on home oxygen. Although we did not observe differences in respiratory support duration, reduced home oxygen may indicate less severe disease and is an important outcome for families.

We did observe increased NEC in the post-QIC. There is no clear physiological rationale for increased risk of NEC with the respiratory interventions introduced. Meta-analyses of DCC, MIST, and CPAP have not found any association between these interventions and increased NEC [14, $15,28]$. Given the importance of NEC, we reviewed rates for infants $<32$ week gestation admitted to our unit in the entire calendar years of 2018 and 2019 (including our pre- and post-QI periods), finding the rates were not significantly different over these longer sample periods. NEC is recognised to occur in temporal clusters, which could explain this finding in the absence of another likely explanation. However, ongoing monitoring of this clinically important outcome in our population will continue.

Several factors contributed to the success of this QI initiative. We identified drivers impacting BPD incidence and developed an evidence-based bundle specific to our population. The relatively large number of VPTIs born at $\mathrm{MCH}$ allows us to draw some conclusions from the short duration of a rapid-cycle QI process. Ongoing educational support and review of outcomes will allow continuous re-evaluation of practice and identify further areas for improvement. Unlike prior QI studies, our approach incorporated the recently adopted interventions DCC and MIST, showing promising reduction in MV.

There are some limitations to this study, including the use of historical controls. There is potential for confounding due to secular trends and changes to NICU practice. We can report temporal associations but cannot conclude that practice change alone was responsible for the observed outcomes. Ongoing training and culture shift towards less invasive ventilation may have resulted in improved processes, rather than the interventions alone. When analysing results, QI bundles are viewed as one intervention with multiple synergistic components. Therefore, we are unable to ascertain the individual impact of each component of the QI bundle. Finally, our sample size is not sufficient to draw conclusions on less common outcomes such as BPD and mortality. We will continue to monitor these outcomes in future QI cycles.

Focus for planned future QI cycles in our centre includes increasing ANS administration, improving documentation, and introducing a standardised extubation protocol and video-recorded resuscitation review. We hope that in time, reductions in MV will be further translated into reductions in BPD in our patient population.

In our rapid cycle QI study, we demonstrated implementation of a QI respiratory bundle consisting of DCC, early nasal bCPAP, and MIST in VPTIs to be associated with reduced rates of $\mathrm{MV}$ in the DR, the first $72 \mathrm{~h}$ of life, and during admission, and in home oxygen treatment. The increased rate of NEC that we observed, although not sustained in a larger sample, illustrates the importance of monitoring outcomes after QI interventions for unexpected results, especially when multiple interventions are bundled. Further QI studies incorporating DCC and MIST would be of value to establish external validity in other centres.

\section{Statement of Ethics}

The study was approved by Monash Health Human Research Ethics Committee, Melbourne (RES-18-0000-726L), and Monash University Human Research Ethics Committee, Melbourne (18941). This was deemed a low-risk project; thus, informed consent from parents was not required.

\section{Conflict of Interest Statement}

The authors have no conflicts of interest to declare.

\section{Funding Sources}

C.T.R. is supported by a National Health and Medical Research Council (Australia) Investigator Grant (1175634).

\section{Author Contributions}

S.C.-Y.L., C.T.R., and R.B. conceptualised and designed the study, interpreted the data, and revised and approved the final manuscript. S.C.-Y.L. performed the data collection and analysis and drafted the initial manuscript; she is the guarantor.

\section{Data Availability Statement}

All data generated or analysed during this study are included in this article and its online suppl. files. Further enquiries can be directed to the corresponding author. 


\section{References}

1 Cheong JLY, Doyle LW. An update on pulmonary and neurodevelopmental outcomes of bronchopulmonary dysplasia. Semin Perinatol. 2018;42:478-84.

2 Jensen EA, Dysart K, Gantz MG, McDonald S, Bamat NA, Keszler M, et al. The diagnosis of bronchopulmonary dysplasia in very preterm infants: an evidence-based approach. Am J Respir Crit Care Med. 2019;200(6):7519.

3 Anderson PJ, Doyle LW. Neurodevelopmental outcome of bronchopulmonary dysplasia. Semin Perinatol. 2006;30:227-32.

4 Doyle LW, Carse E, Adams AM, Ranganathan S, Opie G, Cheong JLY, et al. Ventilation in extremely preterm infants and respiratory function at 8 years. N Engl J Med. 2017;377: 329-37.

5 Jobe AH. Mechanisms of lung injury and bronchopulmonary dysplasia. Am J Perinatol. 2016;33:1076-8.

6 AIHW. Australia's mothers and babies 2016 - in brief. Canberra: Australian Institute of Health and Welfare; 2018. p. 34.

7 Chow SSW, Creighton P, Chambers GM, Lui K. Report of the Australian and New Zealand neonatal network 2016. Sydney: ANZNN; 2018.

8 Birenbaum HJ, Dentry A, Cirelli J, Helou S, Pane MA, Starr K, et al. Reduction in the incidence of chronic lung disease in very low birth weight infants: results of a quality improvement process in a tertiary level neonatal intensive care unit. Pediatrics. 2009;123:4450.

9 Kakkilaya V, Jubran I, Mashruwala V, Ramon E, Simcik VN, Marshall M, et al. Quality improvement project to decrease delivery room intubations in preterm infants. Pediatrics. 2019;143(1):e20180201.

10 Kubicka Z, Zahr E, Rousseau T, Feldman HA, Fiascone J. Quality improvement to reduce chronic lung disease rates in very-low birth weight infants: high compliance with a respiratory care bundle in a small NICU. J Perinatol. 2018;38:285-92.
11 Levesque BM, Kalish LA, LaPierre J, Welch $\mathrm{M}$, Porter V. Impact of implementing 5 potentially better respiratory practices on neonatal outcomes and costs. Pediatrics. 2011; 128:e218-26.

12 Wlodaver A, Blunt M, Satnes K, Escobedo M, Hallford G, Szyld E. A retrospective comparison of VLBW outcomes before and after implementing new delivery room guidelines at a regional tertiary care center. J Perinatol. 2016; 36:182-5.

13 DeMauro SB, Douglas E, Karp K, Schmidt B, Patel J, Kronberger A, et al. Improving delivery room management for very preterm infants. Pediatrics. 2013;132:e1018-25.

14 Fogarty M, Osborn DA, Askie L, Seidler AL, Hunter K, Lui K, et al. Delayed vs early umbilical cord clamping for preterm infants: a systematic review and meta-analysis. Am J Obstet Gynecol. 2018;218:1-18.

15 Foglia EE, Jensen EA, Kirpalani H. Delivery room interventions to prevent bronchopulmonary dysplasia in extremely preterm infants. J Perinatol. 2017;37:1171-9.

16 Finer NN, Rich W, Wang C, Leone T. Airway obstruction during mask ventilation of very low birth weight infants during neonatal resuscitation. Pediatrics. 2009;123:865-9.

17 Schilleman K, Witlox RS, Lopriore E, Morley CJ, Walther FJ, te Pas AB. Leak and obstruction with mask ventilation during simulated neonatal resuscitation. Arch Dis Child Fetal Neonatal Ed. 2010;95:F398-402.

18 Schmolzer GM, Dawson JA, Kamlin CO, O'Donnell CP, Morley CJ, Davis PG. Airway obstruction and gas leak during mask ventilation of preterm infants in the delivery room. Arch Dis Child Fetal Neonatal Ed. 2011;96: F254-7.

19 Wang TF, Dang D, Liu JZ, Du JF, Wu H. Bubble CPAP for preterm infants with respiratory distress: a meta-analysis. HK J Paediatr. 2016; 21:86-92.
20 Kahn DJ, Habib RH, Courtney SE. Effects of flow amplitudes on intraprong pressures during bubble versus ventilator-generated nasal continuous positive airway pressure in premature infants. Pediatrics. 2008;122:1009-13.

21 Green EA, Dawson JA, Davis PG, De Paoli AG, Roberts CT. Assessment of resistance of nasal continuous positive airway pressure interfaces. Arch Dis Child Fetal Neonatal Ed. 2019;104:F535-9.

22 Slutsky AS. Lung injury caused by mechanical ventilation. Chest. 1999;116:9S-15S.

23 Morley CJ, Davis PG, Doyle LW, Brion LP, Hascoet JM, Carlin JB, et al. Nasal CPAP or intubation at birth for very preterm infants. $\mathrm{N}$ Engl J Med. 2008;358:700-8.

24 Drevhammar T, Nilsson K, Zetterström H, Jonsson B. Comparison of seven infant continuous positive airway pressure systems using simulated neonatal breathing. Pediatr Crit Care Med. 2012;13:e113-9.

25 Horbar JD, Rogowski J, Plsek PE, Delmore P, Edwards WH, Hocker J, et al. Collaborative quality improvement for neonatal intensive care. NIC/Q Project Investigators of the Vermont Oxford Network. Pediatrics. 2001;107: 14-22.

26 Lee SK, Shah PS, Singhal N, Aziz K, Synnes A, McMillan D, et al. Association of a quality improvement program with neonatal outcomes in extremely preterm infants: a prospective cohort study. CMAJ. 2014;186:E485-94.

27 Walsh M, Laptook A, Kazzi SN, Engle WA, Yao Q, Rasmussen M, et al. A cluster-randomized trial of benchmarking and multimodal quality improvement to improve rates of survival free of bronchopulmonary dysplasia for infants with birth weights of less than 1250 grams. Pediatrics. 2007;119:876-90.

28 Schmolzer GM, Kumar M, Pichler G, Aziz K, O'Reilly M, Cheung PY. Non-invasive versus invasive respiratory support in preterm infants at birth: systematic review and metaanalysis. BMJ. 2013;347:f5980. 